\title{
SBC2007-176341
}

\section{A POROVISCOELASTIC MODEL OF INTRALUMINAL THROMBUS FROM ABDOMINAL AORTIC ANEURYSMS}

\author{
Federica Boschetti (1), Elena Di Martino (2), Giancarlo Gioda (3)
}

\author{
(1) Laboratory of Biological Structure Mechanics, \\ Department of Structural Engineering, \\ Politecnico di Milano, ITALY
}

(2) Institute for Complex Engineered Systems, Carnegie Mellon University, Pittsburgh PA, USA

\section{(3) Department of Structural Engineering, Politecnico di Milano, ITALY}

\section{INTRODUCTION}

Computational models, developed to study abdominal aortic aneurysm (AAA) biomechanics, demonstrated that the presence of an intraluminal thrombus (ILT) can significantly alter the wall stress distribution in the degenerated vessel wall [1,2]. ILT is a soft hydrated tissue constituted of $90 \%$ of water. The solid porous matrix is made of a fibrin lattice entrapping solid aggregates, mostly platelets [3].. Although the ILT is subjected to compression in vivo, up to now ILT constitutive models have been based on parameters derived from tensile testing of ILT specimens [1]. The aim of this study was to define the biomechanical properties of ILT under compressive loads, using a combined experimental-numerical approach. ILT samples were tested for unconfined compression and permeation. A poroviscoelastic model was implemented to interpret the experimental data.

\section{METHODS}

\section{Biomechanical tests}

From each ILT, removed from patients undergoing surgical AAA resection, we obtained several samples representing different transmural layers. Specimens were cylindrically shaped (diameter $7 \mathrm{~mm}$, height $1 \mathrm{~mm}$ ).

For different samples from each ILT we performed step wise stress-relaxation unconfined compression using the ELF3200 machine (Bose Corporation, Eden Prairie, MN, USA) equipped with a $22 \mathrm{~N}$ load cell, and permeation tests, using a custom made device. The thickness of the specimens was measured from the position of the testing machine actuator, after imposing a preload of $0.02 \mathrm{~N}$. The stepwise compression was applied, after a pre-compression to $40 \%$, as a series of 3 strain ramps, each $5 \%$ of the initial thickness, at a velocity of $10 \mu \mathrm{m} / \mathrm{s}$, followed by 2 hours of stress relaxation to equilibrium. The elastic modulus was calculated for the applied increasing strain levels from the equilibrium stress-relaxation response. The Darcy permeability was calculated by measuring the flow through a sample subjected to a fluid pressure head of $100 \mathrm{mmHg}$.

\section{Numerical model}

The analysis of the stress-relaxation test was based on an axisymmetric finite element model consisting of 600 quadrilateral, 8 node isoparametric elements. To allow for the effects of consolidation the nodal variables are represented by the displacement components of the solid phase and by the neutral pressure of the liquid phase. In order to avoid possible inconsistencies, caused by different variations of the effective and neutral stresses within the elements, the pore pressure was defined solely at the 4 corner nodes of each element.

The viscous behaviour of the solid phase is governed by a Kelvin model (Fig. 1) through the following constitutive equation

$$
\underline{\sigma}^{\prime}(t)=\underline{D}^{v e} \cdot \underline{\varepsilon}^{v e}(t)+\underline{V}^{v e} \cdot \underline{\dot{\varepsilon}}^{v e}(t)
$$

where $\sigma$ is the effective stress vector acting on the solid phase, $\varepsilon^{v e}$ is the visco-elastic strain vector, $\underline{D}^{v e}$ and $\underline{V}^{v e}$ are, respectively, the elastic and viscous constitutive matrices.

Under the assumption of isotropy, the six mechanical parameters of the rheological model consist of the shear and bulk moduli of the first elastic element $\left(G_{E}\right.$ and $B_{E}$, or $E$ and $\left.v\right)$, which govern the instantaneous response of the model, of the shear and bulk moduli of the second elastic element of the model $\left(G_{V}\right.$ and $\left.B_{V}\right)$ and of the corresponding shear and bulk viscosity coefficients ( $\eta_{\mathrm{BV}}$ and $\eta_{\mathrm{GV}}$ ). The volumetric viscous behaviour is neglected; therefore the number of parameters reduces to 4 . 


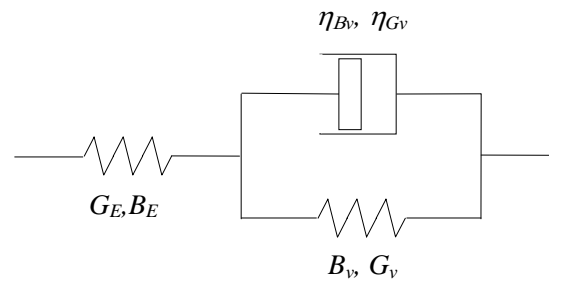

Figure 1. Kelvin model and its parameters

This material model, including the hydraulic conductivity as an additional material parameter, $\mathrm{k}$, was introduced in the finite element equations that govern the Biot consolidation problem [4,5], that for the linear elastic case read

$$
\left[\begin{array}{cc}
\underline{0} & \underline{0} \\
\underline{0} & -\frac{1}{\gamma_{w}} \underline{M}
\end{array}\right] \cdot\left\{\begin{array}{l}
\underline{u} \\
\underline{p}
\end{array}\right\}+\left[\begin{array}{cc}
\underline{K} & \underline{C} \\
\underline{C}^{T} & -\underline{S}_{w}
\end{array}\right] \cdot\left\{\begin{array}{l}
\underline{\dot{u}} \\
\dot{\dot{p}}
\end{array}\right\}=\left\{\begin{array}{l}
\underline{\dot{f}} \\
\underline{0}
\end{array}\right\}
$$

In Eq.(2) $\underline{u}$ and $\underline{p}$ are the vectors of nodal displacements and neutral pressures, $\gamma_{w}$ is the unit weight of the liquid phase, $\underline{M}$ and $K$ are respectively the flow and the stiffness matrices, $\underline{C}$ is the matrix coupling the nodal pore pressures to the nodal forces, $\underline{S}_{w}$ is the matrix representing the volumetric deformability of the liquid phase, $f$ is the nodal force vector and a superposed dot means time derivative.

\section{RESULTS AND DISCUSSION}

Mechanical tests were performed on 49 specimens from 8 ILTs obtained fresh from discarded operating room tissue. Average values are shown in Figure 2. Results show that the mechanical properties vary widely across the thickness suggesting a stiffer structure moving from the luminal to the abluminal layer. The permeability results higher for the medial layer. SEM images from one ILT indeed reveal a non homogeneous structure with varying pore size through the thickness (Figure 3). This stratification of the material properties may be related to ILT formation which occurs in time by successive layers addition.
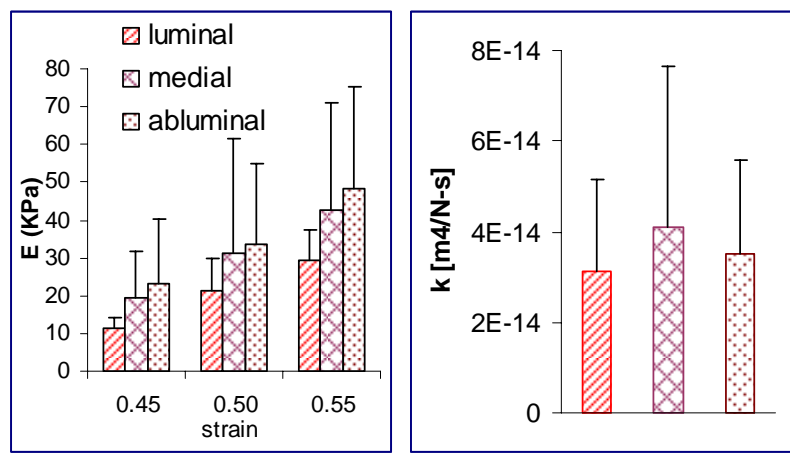

Figure 2. Elastic modulus for increasing strain levels (left), and Darcy permeability at $100 \mathrm{mmHg}$ (right), both at different locations through the ILT thickness. Data from 8 ILTs.

The visco-elastic consolidation program written was used for simulating one step of the stress-relaxation unconfined test. Upon calibration of the mentioned material parameters, and of the coefficient of hydraulic conductivity, the finite element model led to the time-load curve that is compared in Figure 4 with the experimental results. The model parameters were set as strain dependent in order to obtain a good fit between measured and computed curves. In particular, the elastic parameter $\mathrm{E}$ and the hydraulic conductivity $\mathrm{k}$ were changed between compression steps, whereas the viscosity parameter $\eta_{\mathrm{GV}}$ was changed even within each step as a function of the computed viscous strain.

The agreement between the two sets of data is encouraging and suggests that the approach taken to simulate the viscoelasticity of the ILT is valid.
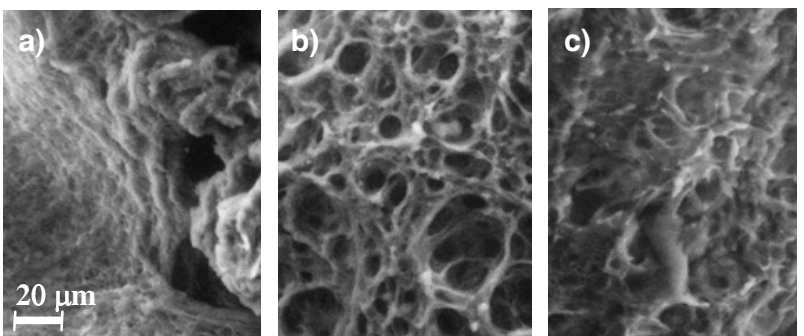

Figure 3. SEM images (Zeiss, EVO50 EP) of a ILT section showing a porous structure with different pore sizes. a) Iuminal; b) medial; c) abluminal

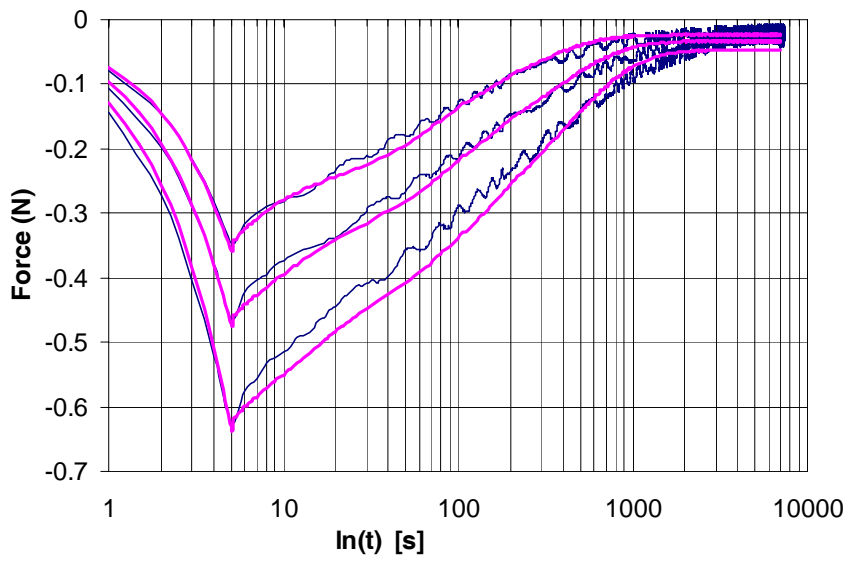

Figure 4. Comparison between the measured (blue, thin line) and computed (magenta, thick line) force of a stepwise stress relaxation experiment.

\section{REFERENCES}

1. Wang D.H., Makaroun M., Webster M.W., and Vorp D.A., 2001, "Mechanical Properties and Microstructure of Intraluminal Thrombus from Abdominal Aortic Aneurysm", Journal of Biomechanical Engineering 123(6), pp. 536-539.

2. Di Martino E.S., and Vorp D.A., 2003, "Effect of Variation in Intraluminal Thrombus Constitutive Properties on Abdominal Aortic Aneurysm Wall Stress", Annals of Biomedical Engineering, 31(7), pp. 804-809.

3. Adolph R., Vorp D.A., Steed D.L., Webster M.W., 1997, "Cellular Content and Permeability of Intraluminal Thrombus in Abdominal Aortic Aneurysm", J. Vasc. Surg. 25, pp. 916-926.

4. Biot M.A., 1941, "General Theory of Three-Dimensional Consolidation", Journal of Applied Physics 12, pp. 55-164.

5. Zienkiewicz O.C., 1977, The Finite Element Method. McGraw-Hill, London. 